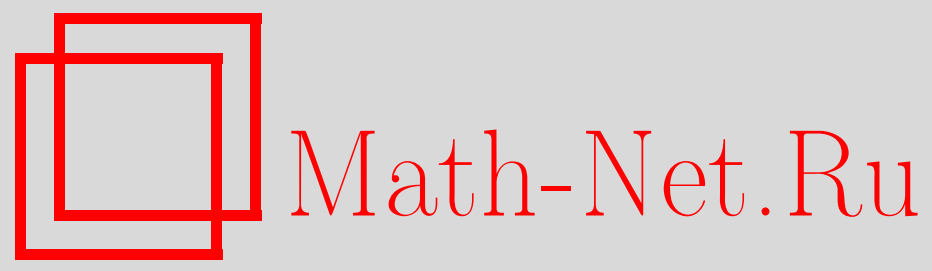

Д. С. Широков, Использование обобщённой теоремы Паули для нечётных элементов алгебры Клиффорда для анализа связей между спинорными и ортогональными группами произвольных размерностей, Вестн. Сам. гос. техн. ун-та. Сер. Физ.-мат. науки, 2013, выпуск 1(), 279-287

DOI: https://doi.org/10.14498/vsgtu1176

Использование Общероссийского математического портала Math-Net.Ru подразумевает, что вы прочитали и согласны с пользовательским соглашением

http://www.mathnet.ru/rus/agreement

Параметры загрузки:

IP : 34.227 .88 .159

26 апреля 2023 г., 09:52:01

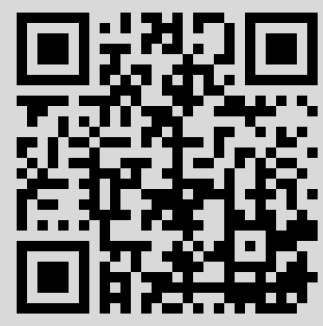


УДК 514.744

\title{
ИСПОЛЬЗОВАНИЕ ОБОБЩЁННОЙ ТЕОРЕМЫ ПАУЛИ ДЛЯ НЕЧЁТНЫХ ЭЛЕМЕНТОВ АЛГЕБРЫ КЛИФФОРДА ДЛЯ АНАЛИЗА СВЯЗЕЙ МЕЖДУ СПИНОРНЫМИ И ОРТОГОНАЛЬНЫМИ ГРУППАМИ ПРОИЗВОЛЬНЫХ РАЗМЕРНОСТЕЙ
}

\section{Д. С. Широков}

Математический институт им. В.А. Стеклова РАН, Россия, 119991, Москва, ул. Губкина, 8.

E-mail: shirokov@mi.ras.ru

\begin{abstract}
С помощъю обобщённой теоремъ Паули доказывается теорема о двулистном накрытии ортогональных групп спинорными. Доказанъ теоремъ о двулистных накрытиях ортохронной, ортохорной, специальной и специальной ортохронной групп соответствующими спинорными группами. Показано различие подходов с использованием присоединённого действия и изменённого присоединенного действия.
\end{abstract}

Ключевые слова: алгебры Клиффорда, теорема Паули, спинорнъе группь, ортогональные группь, теорема о двулистном накрытии, ортохронная группа, ортохорная группа.

1. Вещественные и комплексные алгебры Клиффорда. Рассмотрим вещественную $(\mathbb{F}=\mathbb{R})$ или комплексную $(\mathbb{F}=\mathbb{C})$ алгебру Клиффорда $\mathcal{C} \ell^{\mathbb{F}}(p, q)$ (см. подробнее в предыдущей работе автора [2]), задаваемую набором генераторов $e^{a}, a=1,2, \ldots, n$. Генераторы удовлетворяют определяющим антикоммутационным соотношениям алгебры Клиффорда $e^{a} e^{b}+e^{b} e^{a}=2 \eta^{a b} e$, где $\eta=\left\|\eta^{a b}\right\|=\operatorname{diag}(1, \ldots, 1,-1, \ldots,-1)$ есть диагональная матрица, у которой $p$ штук 1 и $q$ штук -1 на диагонали. Базисные элементы $e^{A}$ занумерованы упорядоченными мультииндексами $A$ длины от 0 до $n$. Произвольный элемент алгебры Клиффорда будем записывать как

$$
U=u e+u_{a} e^{a}+\sum_{a_{1}<a_{2}} u_{a_{1} a_{2}} e^{a_{1} a_{2}}+\ldots+u_{1 \ldots n} e^{1 \ldots n}=u_{A} e^{A}, \quad u_{A} \in \mathbb{F} \quad \forall A .
$$

Векторные подпространства, натянутые на элементы $e^{a_{1} \ldots a_{k}}$, занумерованные упорядоченными мультииндексами длины $k$, обозначаются через $\mathcal{C} \ell_{k}^{\mathbb{F}}(p, q)$ и называются подпространствами элементов ранга $k$. Алгебра Клиффорда $\mathcal{C} \ell^{\mathbb{F}}(p, q)$ является супералгеброй, а именно представляется в виде прямой суммы чётного и нечётного подпространств

$$
\mathcal{C} \ell^{\mathbb{F}}(p, q)=\mathcal{C} \ell_{\text {Even }}^{\mathbb{F}}(p, q) \oplus \mathcal{C} \ell_{\text {Odd }}^{\mathbb{F}}(p, q)=\bigoplus_{k-\text { even }} \mathcal{C} \ell_{k}^{\mathbb{F}}(p, q) \oplus \bigoplus_{k-\text { odd }} \mathcal{C} \ell_{k}^{\mathbb{F}}(p, q) .
$$

Рассмотрим две линейные операции сопряжения на алгебре Клиффорда $\mathcal{C} \ell^{\mathbb{F}}(p, q)$. Операция чётностного сопряжения переводит чётные элементы алгебры Клиффорда в себя, а у нечётных элементов меняет знак: $U^{\curlywedge}=\left(U_{\text {even }}+\right.$ $\left.+U_{\text {odd }}\right)^{\curlywedge}=U_{\text {even }}-U_{\text {odd }}$. Операция реверс меняет порядок генераторов в произведении: $\left(\lambda e^{a_{1} \ldots a_{k}}\right)^{\sim}=\lambda e^{a_{k}} \ldots e^{a_{1}}, \lambda \in \mathbb{F}$.

Дмитрий Сергеевич Широков, аспирант, отд. математической физики. 
2. Обобщённая теорема Паули для нечётных элементов алгебры Клиффорда. В [1] автором были предложены обобщения теоремы Паули на случай вещественных и комплексных алгебр Клиффорда. В настоящем изложении рассмотрим случай, когда в качестве двух наборов элементов $\gamma^{a}$ и $\beta^{a}$, удовлетворяющих антикоммутационным соотношениям алгебры Клиффорда, выступают нечётные элементы алгебры Клиффорда. Заметим, что в отличие от общего случая [1] мы имеем 2 (а не 6) различных варианта связи двух наборов элементов алгебры Клиффорда нечётной размерности $n$.

Теорема. Пусть $\mathcal{C} \ell^{\mathbb{F}}(p, q)$ - вещественная (или комплексная) алгебра Клифборда размерности $n=p+q$. Пусть два набора нечётных элементов алгебры Клифборда $\gamma^{a}, \beta^{a} \in \mathcal{C} \ell_{\text {Odd }}^{\mathbb{F}}(p, q), a=1,2, \ldots, n$ удовлетворяют соотношениям $\gamma^{a} \gamma^{b}+\gamma^{b} \gamma^{a}=2 \eta^{a b} e, \beta^{a} \beta^{b}+\beta^{b} \beta^{a}=2 \eta^{a b} e$.

Тогда оба набора генерируют базисы алгебры Клифборда и выражения $\gamma^{1 \ldots n}, \beta^{1 \ldots n}$ принимают значения $\pm e^{1 \ldots n}$. Кроме того:

1) В случае чётного п существует единственный, с точностъю до умножения на ненулевое вещественное (соответственно комплексное) число, обратимый элемент алгебры Клифборда $T\left(\right.$ причём $T \in \mathcal{C} \mathcal{E}_{\mathrm{Even}}^{\mathbb{F}}(p, q)$, если $\beta^{1 \ldots n}=\gamma^{1 \ldots n}, u T \in \mathcal{C} \ell_{\text {Odd }}^{\mathbb{F}}(p, q)$, если $\left.\beta^{1 \ldots n}=-\gamma^{1 \ldots n}\right)$ maкой, что

$$
\gamma^{a}=T^{-1} \beta^{a} T, \quad \forall a=1, \ldots, n .
$$

Кроме того, такой элемент $T$ имеет вид $T=\beta^{A} F \gamma_{A}$, где $F-$ такой элемент из множества $\left\{\gamma^{A}, A \in \mathcal{I}_{\text {Even }}\right\}$, если $\beta^{1 \ldots n}=\gamma^{1 \ldots n}$, и из $\left\{\gamma^{A}, A \in \mathcal{I}_{\text {Odd }}\right\}$, если $\beta^{1 \ldots n}=-\gamma^{1 \ldots n}$, ито $\beta^{A} F \gamma_{A} \neq 0$.

2) В случае нечётного $n$ существует единственный, с точностью до умножения на обратимый элемент иентра, обратимый элемент алгебрьь Клифборда $T \in \mathcal{C} \ell_{\text {Even }}^{\mathbb{F}}(p, q)$ ( а значит, и другой $T \in \mathcal{C} \ell_{\text {Odd }}^{\mathbb{F}}(p, q)$, полученный из первого умножсенем на $\left.e^{1 \ldots n}\right)$ такой, что

$$
\begin{aligned}
& \gamma^{a}=T^{-1} \beta^{a} T, \quad \forall a=1, \ldots, n \quad \Longleftrightarrow \quad \beta^{1 \ldots n}=\gamma^{1 \ldots n}, \\
& \gamma^{a}=-T^{-1} \beta^{a} T, \quad \forall a=1, \ldots, n \quad \Longleftrightarrow \quad \beta^{1 \ldots n}=-\gamma^{1 \ldots n} .
\end{aligned}
$$

При этом в обоих случаях элемент Т имеет вид

$$
T=\sum_{A \in \mathcal{I}_{\text {Even }}} \beta^{A} F \gamma_{A},
$$

где $F$ - такой элемент из множества $\left\{\gamma^{A}, A \in \mathcal{I}_{\text {Even }}\right\}$ (или из множества $\left.\left\{\gamma^{A}, A \in \mathcal{I}_{\text {Odd }}\right\}\right)$, что

$$
\sum_{A \in \mathcal{I}_{\text {Even }}} \beta^{A} F \gamma_{A} \neq 0 .
$$

Также нас будет интересовать связь двух наборов элементов вида $\gamma^{a}=$ $=\left(T^{\curlywedge}\right)^{-1} \beta^{a} T$, где $\curlywedge-$ чётностное сопряжение.

Теорема. При предположениях предыдущей теоремы верно следующее. В случае алгебры Клифборда $\mathcal{C} \ell^{\mathbb{F}}(p, q)$ произвольной (чётной и нечётной) 
размерности п существует единственный, с точностью до умножения на ненулевое вещественное (соответственно комплексное) число, обратимый элемент алгебры Клифборда $T\left(\right.$ причём $T \in \mathcal{C} \mathbb{E}_{\text {Even }}^{\mathbb{F}}(p, q)$, если $\beta^{1 \ldots n}=\gamma^{1 \ldots n} u$ $T \in \mathcal{C} \ell_{\text {Odd }}^{\mathbb{F}}(p, q)$, если $\left.\beta^{1 \ldots n}=-\gamma^{1 \ldots n}\right)$ maкой, что

$$
\gamma^{a}=T^{\curlywedge-1} \beta^{a} T, \quad \forall a=1, \ldots, n .
$$

Кроме того:

1) $B$ случае чётного $n$ такой элемент $T$ имеет вид $T=\beta^{A} F \gamma_{A}$, если $\beta^{1 \ldots n}=\gamma^{1 \ldots n}$, и среди элементов $T=(-1)^{|A|} \beta^{A} F \gamma_{A}$, если $\beta^{1 \ldots n}=$ $=-\gamma^{1 \ldots n}$, где $F-$ такой элемент из множества $\left\{\gamma^{A}, A \in \mathcal{I}_{\text {Even }}\right\}$, если $\beta^{1 \ldots n}=\gamma^{1 \ldots n}$, u из $\left\{\gamma^{A}, A \in \mathcal{I}_{\text {Odd }}\right\}$, если $\beta^{1 \ldots n}=-\gamma^{1 \ldots n}$, что построенный по нему $T$ отличен от нуля.

2) В случае нечётного $n$ такой элемент $T$ имеет вид

$$
T=\sum_{A \in \mathcal{I}_{\text {Even }}} \beta^{A} F e_{A},
$$

где $F$ - такой элемент из множества $\left\{\gamma^{A}, \quad A \in \mathcal{I}_{\text {Even }}\right\}$, если $\beta^{1 \ldots n}=$ $=\gamma^{1 \ldots n}, u$ из $\left\{\gamma^{A}, \quad A \in \mathcal{I}_{\text {Odd }}\right\}$, если $\beta^{1 \ldots n}=-\gamma^{1 \ldots n}$, чmо

$$
\sum_{A \in \mathcal{I}_{\text {Even }}} \beta^{A} F e_{A} \neq 0 .
$$

Заметим, что в последней теореме элемент $T$, о существовании которого идёт речь, единственен с точностью до умножения на произвольную ненулевую константу (а не на произвольный обратимый элемент центра, который в случае нечётной размерности представляет собой элемент из подпространства $\left.\mathcal{C} \ell_{0}^{\mathbb{F}}(p, q) \oplus \mathcal{C} \ell_{n}^{\mathbb{F}}(p, q)\right)$.

3. Связь группы Липшица и ортогональных групп. Рассмотрим $п р и с о е д и-$ нённое действие

$$
\text { ad }: \mathcal{C} \ell^{\mathbb{R} \times}(p, q) \rightarrow \operatorname{End} C \ell^{\mathbb{R}}(p, q),
$$

действующее на группе обратимых элементов вещественной алгебры Клиффорда $\mathcal{C} \ell^{\mathbb{R} \times}(p, q)$ как $T \mapsto \operatorname{ad}_{T}$, где $\operatorname{ad}_{T} U=T U T^{-1}$ для любого $U \in \mathcal{C} \ell^{\mathbb{R}}(p, q)$.

Рассмотрим измененное (twisted) присоединенное действие

$$
\stackrel{\text { ad }}{ }: \mathcal{C} \ell^{\mathbb{R} \times}(p, q) \rightarrow \operatorname{End} C \ell^{\mathbb{R}}(p, q)
$$

которое задаётся как $T \mapsto \curlywedge_{\mathrm{ad}}$, где $\mathrm{ad}_{T} U=T^{\curlywedge} U T^{-1}$ для любого $U \in \mathcal{C} \ell^{\mathbb{R}}(p, q)$.

Рассмотрим әруппу Липшиц, ${ }^{1}$

$$
\Gamma^{ \pm}(p, q)=\left\{T \in \mathcal{C} \ell_{\text {Even }}^{\mathbb{R} \times}(p, q) \cup \mathcal{C} \ell_{\text {Odd }}^{\mathbb{R} \times}(p, q) \mid \forall x \in \mathcal{C} \ell_{1}^{\mathbb{R}}(p, q), T x T^{-1} \in \mathcal{C} \ell_{1}^{\mathbb{R}}(p, q)\right\}
$$

и её специальную подгруппу $\Gamma^{+}=\left\{T \in \Gamma^{ \pm}(p, q) \mid T \in \mathcal{C} \ell_{\text {Even }}^{\mathbb{R} \times}(p, q)\right\}$.

\footnotetext{
13десь и далее символом × обозначается взятие подмножества обратимых элементов соответствующего множества.
} 
В случае алгебры Клиффорда чётной размерности $n$ гомоморфизмы ad и ad сюръективно отображают группу Липшица $\Gamma^{ \pm}$в псевдоортогональную группу $\mathrm{O}(p, q)=\left\{A \in \operatorname{Mat}(n, \mathbb{R}) \mid A^{\top} \eta A=\eta\right\}$. В случае алгебры Клиффорда нечётной размерности $n$ утверждение будет верно только для гомоморфизма ad. Эти факты в литературе доказываются с применением теоремы Картана-Дьедонне [3]. Далее мы докажем эти факты другим путём, с использованием обобщённой теоремы Паули (и без использования теоремы КартанаДьедонне). Мы будем также рассматривать специальную подгруппу псевдоортогональной группы $\mathrm{SO}(p, q)=\{A \in \mathrm{O}(p, q) \mid \operatorname{det} A=1\}$.

Теорема. Рассмотрим алгебру Клифборда $\mathcal{C} \ell^{\mathbb{R}}(p, q)$ размерности $n=p+q$. Тогда следующие отображения сторгективны с ядром $\mathcal{C} \ell_{0}^{\mathbb{R} \times}(p, q)$ :

$$
\begin{aligned}
& \operatorname{ad}\left(\Gamma^{ \pm}\right)=\mathrm{O}(p, q) \quad \text { при чётном } n ; \\
& \hat{\operatorname{ad}}\left(\Gamma^{ \pm}\right)=\mathrm{O}(p, q), \quad \hat{\operatorname{ad}}\left(\Gamma^{+}\right)=\operatorname{ad}\left(\Gamma^{+}\right)=\mathrm{SO}(p, q) \text {. }
\end{aligned}
$$

Следующее отображсение сюоргективно с ядром $\left\{\mathcal{C} \ell_{0}^{\mathbb{R} \times}(p, q), \mathcal{C} \ell_{n}^{\mathbb{R} \times}(p, q)\right\}$ :

$$
\operatorname{ad}\left(\Gamma^{ \pm}\right)=\operatorname{SO}(p, q) \quad \text { при нечётном } n .
$$

Доказ ат ель ст в о. Возьмём произвольную псевдоортогональную матрицу $P=\left\|p_{b}^{a}\right\| \in \mathrm{O}(p, q)$, и построим набор элементов $\beta^{a}=p_{b}^{a} e^{b}$. Легко проверить, что набор $\left\{\beta^{a}\right\}$ удовлетворяет определяющим соотношениям алгебры Клиффорда $\beta^{a} \beta^{b}+\beta^{b} \beta^{a}=p_{c}^{a} p_{d}^{b} e^{c} e^{d}+p_{d}^{b} p_{c}^{a} e^{d} e^{c}=p_{c}^{a} p_{d}^{b} 2 \eta^{c d} e=2 \eta^{a b} e$, т. к. $P^{\top} \eta P=\eta$. Тогда для наборов $\left\{e^{a}\right\}$ и $\left\{\beta^{a}\right\}$ применима обобщённая теорема Паули (см. предыдущий параграф), а именно существует элемент $T \in \mathcal{C} \ell_{\text {Even }}^{\mathbb{R}}(p, q) \cup \mathcal{C} \ell_{\text {Odd }}^{\mathbb{R}}(p, q)$ такой, что $T e^{a} T^{-1}=\beta^{a}=p_{b}^{a} e^{b}$. Из этой формулы следует, что $\forall x \in \mathcal{C} \ell_{1}^{\mathbb{R}}(p, q), T x T^{-1} \in \mathcal{C} \ell_{1}^{\mathbb{R}}(p, q)$. Итак, $T \in \Gamma^{ \pm}$и мы получили соответствие, при котором для каждой матрицы $P \in \mathrm{O}(p, q)$ найдется элемент из группы $\Gamma^{ \pm}$.

Имеем $\beta^{1 \ldots n}=p_{a_{1}}^{1} e^{a_{1}} p_{a_{2}}^{2} e^{a_{2}} \ldots p_{a_{n}}^{n} e^{a_{n}}=(\operatorname{det} P) e^{1 \ldots n}$. В последнем выражении коэффициенты при всех элементах базиса, отличных от $e^{1 \ldots n}$, равны нулю. Чтобы показать это, надо воспользоваться тем, что $\beta^{1 \ldots n}= \pm e^{1 \ldots n}$.

Заключаем, что если $\beta^{1 \ldots n}=e^{1 \ldots n}($ т. е. $\operatorname{det} P=1)$, то $T \in \mathcal{C} \ell_{\curlywedge}^{\mathbb{R}} \operatorname{lven}(p, q)$. В противном случае имеем $T \in \mathcal{C} \ell_{\mathrm{Odd}}^{\mathbb{R}}(p, q)$. В случае отображения $\hat{\mathrm{d}}$ пользуемся другой теоремой из предыдущего параграфа.

4. Связь ортогональных и спинорных групп. Заметим, что ядром присоединенного действия $\operatorname{ker}(\mathrm{ad})$ является множество $\mathcal{C} \mathbb{R}_{0}^{\mathbb{R} \times}(p, q)$ в случае чётного $n$ и подпространство $\left(\mathcal{C} \ell_{0}^{\mathbb{R}}(p, q) \oplus \mathcal{C} \ell_{n}^{\mathbb{R}}(p, q)\right)^{\times}$в случае нечётного $n$. Ядром изменённого присоединенного действия $\operatorname{ker}(\hat{\mathrm{ad}})$ является множество $\mathcal{C} \ell_{0}^{\mathbb{R} \times}(p, q)$ в случае произвольного $n$.

Рассмотрим на алгебре Клиффорда $\mathcal{C} \ell^{\mathbb{R}}(p, q)$ гомоморфизм $N: \mathcal{C} \ell^{\mathbb{R}}(p, q) \rightarrow$ $\mathcal{C} \ell^{\mathbb{R}}(p, q)$, задаваемый следующим образом: $U \mapsto N(U)=U^{\sim} U$. Говорят, что этот гомоморфизм задает «норму» элементов алгебрь Клиффорда $\mathcal{C} \mathbb{R}^{\mathbb{R}}(p, q)$. Можно также ввести другую «норму» $\stackrel{\curlywedge}{N}: \mathcal{C} \ell^{\mathbb{R}}(p, q) \rightarrow \mathcal{C} \ell^{\mathbb{R}}(p, q)$, которая задаётся как $U \mapsto \stackrel{\curlywedge}{N}(U)=U^{\sim \curlywedge} U$. 
Лемма. Нормы $N(T)=T^{\sim} T$ u $\stackrel{\curlywedge}{N}(T)=T^{\sim \curlywedge} T$ отображают груnпy Лиnшица $\Gamma^{ \pm}$в множество $\mathcal{C} \ell_{0}^{\mathbb{R} \times}(p, q)$.

Доказ а тельств о. Для любого $x \in \mathcal{C} \mathbb{R}_{1}^{\mathbb{R}}(p, q)$ имеем $\left(T x T^{-1}\right)^{\sim}=T x T^{-1}$, $\left(T x T^{-1}\right)^{\sim}=\left(T^{-1}\right)^{\sim} x T^{\sim}$ и $\left(T^{\sim}\right)^{-1}=\left(T^{-1}\right)^{\sim}$. Тогда $T^{\sim} T x=x T^{\sim} T$, т. е. $T^{\sim} T$ лежит в центре алгебры Клиффорда. Так как $T \in \Gamma^{ \pm}$либо чётный, либо нечётный, то $T^{\sim} T$ - чётный элемент и, следовательно, он является элементом вида $\lambda e$. Так как $T \neq 0$, то $\lambda \neq 0$. Заметим, что $\hat{N}(T)= \pm N(T)$ для $T \in \Gamma^{ \pm}$и знак зависит от чётности элемента $T$.

Нормируя группу Липшица, получаем следующие 5 сnинорных груnn:

$$
\begin{aligned}
\operatorname{Pin}(p, q) & =\left\{T \in \Gamma^{ \pm} \mid T^{\sim} T= \pm e\right\}=\left\{T \in \Gamma^{ \pm} \mid T^{\sim \curlywedge} T= \pm e\right\}, \\
\operatorname{Pin}_{\downarrow}(p, q) & =\left\{T \in \Gamma^{ \pm} \mid T^{\sim} T=+e\right\}, \\
\operatorname{Pin}_{\uparrow}(p, q) & =\left\{T \in \Gamma^{ \pm} \mid T^{\sim \curlywedge} T=+e\right\}, \\
\operatorname{Spin}_{1}(p, q) & =\left\{T \in \Gamma^{+} \mid T^{\sim} T= \pm e\right\}=\left\{T \in \Gamma^{+} \mid T^{\sim \curlywedge} T= \pm e\right\}, \\
\operatorname{Spin}_{\uparrow \downarrow}(p, q) & =\left\{T \in \Gamma^{+} \mid T^{\sim} T=+e\right\}=\left\{T \in \Gamma^{+} \mid T^{\sim \curlywedge} T=+e\right\} .
\end{aligned}
$$

Из определения спинорных групп следует, что группа $\operatorname{Pin}(p, q)$ при $p \neq 0$ и $q \neq 0$ состоит из четырёх (не обязательно связных) компонент (в вырожденных случаях $p=0$ и $q=0$ - из двух компонент):

$$
\operatorname{Pin}(p, q)=\operatorname{Spin}_{\uparrow \downarrow}(p, q) \sqcup \operatorname{Spin}^{\prime}(p, q) \sqcup \operatorname{Pin}_{\uparrow}^{\prime}(p, q) \sqcup \operatorname{Pin}_{\downarrow}^{\prime}(p, q),
$$

где $\operatorname{Pin}_{\uparrow}^{\prime}(p, q)=\operatorname{Pin}_{\uparrow}(p, q) \backslash \operatorname{Spin}_{\uparrow \downarrow}(p, q), \operatorname{Pin}_{\downarrow}^{\prime}(p, q)=\operatorname{Pin}_{\downarrow}(p, q) \backslash \operatorname{Spin}_{\uparrow \downarrow}(p, q)$, $\operatorname{Spin}^{\prime}(p, q)=\operatorname{Spin}(p, q) \backslash \operatorname{Spin}_{\uparrow \downarrow}(p, q)$.

Теорема. Следующие отображения сюргективны с ядром $\{ \pm 1\}$ :

$$
\begin{aligned}
\operatorname{ad}(\operatorname{Pin}(p, q)) & =\mathrm{O}(p, q) \quad \text { при чётном } n ; \\
\curlywedge & \curlywedge \\
\operatorname{ad}(\operatorname{Pin}(p, q)) & =\mathrm{O}(p, q), \quad \text { ad }(\operatorname{Spin}(p, q))=\operatorname{ad}(\operatorname{Spin}(p, q))=\operatorname{SO}(p, q) .
\end{aligned}
$$

Следующее отображение сюргективно с ядром $\left\{ \pm 1, \pm e^{1 \ldots n}\right\}$ :

$$
\text { ad }: \operatorname{Pin}(p, q) \rightarrow \operatorname{SO}(p, q) \quad \text { при нечётном } n \text {. }
$$

Доказ ательств о. Теорема следует из теоремы предыдущего параграфа и леммы.

5. Случай остальных ортогональных групп. Будем рассматривать псевдоевклидово пространство $\mathbb{R}^{p, q}$ сигнатуры $(p, q)$ и называть первые $p$ координат временными, а последние $q$ - пространственными. Будем рассматривать следующие подгруппы псевдоортогональной группы: ортохронную $\mathrm{O}_{\uparrow}(p, q)=$ $=\left\{A \in \mathrm{O}(p, q) \mid A_{1 \ldots p}^{1 \ldots p}>0\right\}$, ортохорную $\mathrm{O}_{\downarrow}(p, q)=\left\{A \in \mathrm{O}(p, q) \mid A_{p+1 \ldots n}^{p+1 \ldots n}>0\right\}$ и специальную ортохронную $\mathrm{SO}_{\uparrow \downarrow}(p, q)=\left\{A \in \mathrm{O}(p, q) \mid A_{1 \ldots p}^{1 \ldots p}>0, \operatorname{det} A=1\right\}$, где $A_{1 \ldots p}^{1 \ldots p}$ и $A_{p+1 \ldots n}^{p+1 \ldots n}$ - соответствующие миноры матрицы $A$ (см. также [2]). Отметим, что ортохронная группа состоит из преобразований псевдоевклидова 
пространства, сохраняющих ориентацию во времени. Ортохорную группу образуют преобразования, сохраняющие пространственную ориентацию. Группа $\mathrm{O}(p, q)$ при $p \neq 0$ и $q \neq 0$ состоит из четырёх связных компонент $\mathrm{O}(p, q)=$ $=\mathrm{SO}_{\uparrow \downarrow}(p, q) \sqcup \mathrm{SO}^{\prime}(p, q) \sqcup \mathrm{O}_{\uparrow}^{\prime}(p, q) \sqcup \mathrm{O}_{\downarrow}^{\prime}(p, q)$, где $\mathrm{O}_{\uparrow}^{\prime}(p, q)=\mathrm{O}_{\uparrow}(p, q) \backslash \mathrm{SO}_{\uparrow \downarrow}(p, q)$, $\mathrm{O}_{\downarrow}^{\prime}(p, q)=\mathrm{O}_{\downarrow}(p, q) \backslash \mathrm{SO}_{\uparrow \downarrow}(p, q), \mathrm{SO}^{\prime}(p, q)=\operatorname{Spin}(p, q) \backslash \mathrm{SO}_{\uparrow \downarrow}(p, q)$.

Далее будут сформулированы утверждения о сюръективных отображениях других спинорных групп $\left(\operatorname{Pin}_{\uparrow}(p, q), \operatorname{Pin}_{\downarrow}(p, q)\right.$ и $\left.\operatorname{Spin}_{\uparrow \downarrow}(p, q)\right)$ на подгруппы псевдоортогональной группы $\left(\mathrm{O}_{\uparrow}(p, q), \mathrm{O}_{\downarrow}(p, q)\right.$ и $\left.\mathrm{SO}_{\uparrow \downarrow}(p, q)\right)$. Для их доказательства нам понадобятся утверждение о норме элементов спинорных групп, которое было сформулировано в [2]. На алгебре Клиффорда $\mathcal{C} \ell^{\mathbb{R}}(p, q)$ можно задать структуру евклидова пространства, т. е. задать операцию скалярного произведения $(U, V)=\operatorname{Tr}\left(U^{\dagger} V\right)$. Скалярное произведение естественным образом порождает норму $\|U\|=\sqrt{\operatorname{Tr}\left(U^{\dagger} U\right)}$. Здесь $\dagger-$ операция эрмитова сопряжения элементов алгебры Клиффорда (см. [4,2]).

Теорема [2]. Пусть элемент алгебры Клифборда Т принадлежит групne $\operatorname{Pin}(p, q)$ и пусть при гомоморбизме аd элемент $T$ переходит в ортогональную матрицу $A \in \mathrm{O}(p, q)$. Тогда норма элемента $T$ свлзана с главными минорами этой матрищъ $A_{1 \ldots p}^{1 \ldots p}, A_{p+1 \ldots n}^{p+1 \ldots n}$ следующим образом:

$$
\|T\|^{2}=\operatorname{Tr}\left(T^{\dagger} T\right)= \begin{cases}A_{1 \ldots p}^{1 \ldots p}=A_{p+1 \ldots n}^{p+1 \ldots n} & \Leftrightarrow T \in \operatorname{Spin}_{\uparrow \downarrow}(p, q), \\ A_{1 \ldots p}^{1 \ldots p}=-A_{p+1 \ldots n}^{p+1 \ldots n} & \Leftrightarrow T \in \operatorname{Pin}_{\uparrow}^{\prime}(p, q), \\ -A_{1 \ldots p}^{1 \ldots p}=A_{p+1 \ldots n}^{p+1 \ldots n} & \Leftrightarrow T \in \operatorname{Pin}_{\downarrow}^{\prime}(p, q), \\ -A_{1 \ldots p}^{1 \ldots p}=-A_{p+1 \ldots n}^{p+1 \ldots n} & \Leftrightarrow T \in \operatorname{Spin}^{\prime}(p, q) .\end{cases}
$$

Теорема. Следующие гомоморфизмы сюргективны с ядром $\{ \pm 1\}$ :

$$
\begin{gathered}
\stackrel{\curlywedge}{\operatorname{ad}: \operatorname{Pin}_{\uparrow}(p, q)} \rightarrow \mathrm{O}_{\uparrow}(p, q), \quad \stackrel{\curlywedge}{\operatorname{ad}: \operatorname{Pin}_{\downarrow}(p, q) \rightarrow \mathrm{O}_{\downarrow}(p, q),} \\
\stackrel{\curlywedge}{\text { ad: }} \operatorname{Spin}_{\uparrow \downarrow}(p, q) \rightarrow \operatorname{SO}_{\uparrow \downarrow}(p, q) .
\end{gathered}
$$

Доказательство. Теорема следует из двух предыдущих в силу $\|T\|^{2} \geqslant 0$.

Связь спинорных и ортогональных групп явно выражается формулой

$$
T^{\curlywedge} e^{a} T^{-1}=p_{b}^{a} e^{b}
$$

которая ставит в соответствие каждой матрице $P=\left\|p_{b}^{a}\right\|$ из соответствующей ортогональной группы $\mathrm{O}(p, q), \mathrm{SO}(p, q), \mathrm{O}_{\uparrow}(p, q), \mathrm{O}_{\downarrow}(p, q), \mathrm{SO}_{\uparrow \downarrow}(p, q)$ пару элементов $\pm T$ из соответствующей спинорной группы $\operatorname{Pin}(p, q), \operatorname{Spin}(p, q)$, $\operatorname{Pin}_{\uparrow}(p, q), \operatorname{Pin}_{\downarrow}(p, q), \operatorname{Spin}_{\uparrow \downarrow}(p, q)$.

Теперь сформулируем аналогичное утверждение о норме элементов спинорных групп в случае действия гомоморфизма ad.

Теорема. Пусть элемент алгебры Клифборда T принадлежст группе $\operatorname{Pin}(p, q)$ и пусть при гомоморфизме ad элемент $T$ переходит в ортогональную матрицу $A \in \mathrm{O}(p, q)$. Тогда норма элемента $T$ связана с главными минорами этой матрищъ $A_{1 \ldots p}^{1 \ldots p}, A_{p+1 \ldots n}^{p+1 \ldots n}$ следующим образом: 
- в случае $p$ - чётное, $q-$ чётное

$$
\|T\|^{2}=\operatorname{Tr}\left(T^{\dagger} T\right)= \begin{cases}A_{1 \ldots p}^{1 \ldots p}=A_{p+1 \ldots n}^{p+1 \ldots n} & \Leftrightarrow T \in \operatorname{Spin}_{\uparrow \downarrow}(p, q), \\ A_{1 \ldots p}^{1 \ldots p}=-A_{p+1 \ldots n}^{p+1 \ldots n} & \Leftrightarrow T \in \operatorname{Pin}_{\uparrow}^{\prime}(p, q), \\ -A_{1 \ldots p}^{1 \ldots p}=A_{p+1 \ldots n}^{p+1 \ldots n} & \Leftrightarrow T \in \operatorname{Pin}_{\downarrow}^{\prime}(p, q), \\ -A_{1 \ldots p}^{1 \ldots p}=-A_{p+1 \ldots n}^{p+1 \ldots n} & \Leftrightarrow T \in \operatorname{Spin}^{\prime}(p, q)\end{cases}
$$

- в случае $p$-нечётное, $q$-нечётное

$$
\|T\|^{2}= \begin{cases}A_{1 \ldots p}^{1 \ldots p}=A_{p+1 \ldots n}^{p+1 \ldots n} & \Leftrightarrow T \in \operatorname{Spin}_{\uparrow \downarrow}(p, q) \\ -A_{1 \ldots p}^{1 \ldots p}=A_{p+1 \ldots n}^{p+1 \ldots n} & \Leftrightarrow T \in \operatorname{Pin}_{\uparrow}^{\prime}(p, q) \\ A_{1 \ldots p}^{1 \ldots p}=-A_{p+1 \ldots n}^{p+1 \ldots n} & \Leftrightarrow T \in \operatorname{Pin}_{\downarrow}^{\prime}(p, q) \\ -A_{1 \ldots p}^{1 \ldots p}=-A_{p+1 \ldots n}^{p+1 \ldots n} & \Leftrightarrow T \in \operatorname{Spin}^{\prime}(p, q)\end{cases}
$$

- в случае $p$-чётное, $q$-нечётное

$$
\|T\|^{2}= \begin{cases}A_{1 \ldots p}^{1 \ldots p}=A_{p+1 \ldots n}^{p+1 \ldots n} & \Leftrightarrow T \in \operatorname{Spin}_{\uparrow \downarrow}(p, q) \cup \operatorname{Pin}_{\uparrow}^{\prime}(p, q), \\ -A_{1 \ldots p}^{1 \ldots p}=-A_{p+1 \ldots n}^{p+1 \ldots n} & \Leftrightarrow T \in \operatorname{Pin}_{\downarrow}^{\prime}(p, q) \cup \operatorname{Spin}^{\prime}(p, q)\end{cases}
$$

- в случае $p$ - нечётное, $q-$ чётное

$$
\|T\|^{2}= \begin{cases}A_{1 \ldots p}^{1 \ldots p}=A_{p+1 \ldots n}^{p+1 \ldots n} & \Leftrightarrow T \in \operatorname{Spin}_{\uparrow \downarrow}(p, q) \cup \operatorname{Pin}_{\downarrow}^{\prime}(p, q), \\ -A_{1 \ldots p}^{1 \ldots p}=-A_{p+1 \ldots n}^{p+1 \ldots n} & \Leftrightarrow T \in \operatorname{Pin}_{\uparrow}^{\prime}(p, q) \cup \operatorname{Spin}^{\prime}(p, q) .\end{cases}
$$

\section{Teopema.}

1) Если $p, q-$ чётные, то следующие гомоморфизмы сюрвективны с ядром $\{ \pm 1\}$ :

$$
\begin{aligned}
\text { ad }: \operatorname{Pin}_{\uparrow}(p, q) \rightarrow & \mathrm{O}_{\uparrow}(p, q), \quad \operatorname{ad}: \operatorname{Pin}_{\downarrow}(p, q) \rightarrow \mathrm{O}_{\downarrow}(p, q), \\
& \text { ad }: \operatorname{Spin}_{\uparrow \downarrow}(p, q) \rightarrow \operatorname{SO}_{\uparrow \downarrow}(p, q) ;
\end{aligned}
$$

2) если $p, q$ - нечётные, то следующие гомоморфизмы сюргективны с яд-

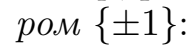

$$
\begin{aligned}
\operatorname{ad}: \operatorname{Pin}_{\uparrow}(p, q) \rightarrow & \mathrm{O}_{\downarrow}(p, q), \quad \operatorname{ad}: \operatorname{Pin}_{\downarrow}(p, q) \rightarrow \mathrm{O}_{\uparrow}(p, q), \\
& \text { ad }: \operatorname{Spin}_{\uparrow \downarrow}(p, q) \rightarrow \mathrm{SO}_{\uparrow \downarrow}(p, q) ;
\end{aligned}
$$

3) если $p$-чётное, $q$-нечётное, то следующие гомоморфизмы сюргективны с соответствующим ядром:

$$
\operatorname{ad}: \operatorname{Pin}_{\uparrow}(p, q) \rightarrow \mathrm{SO}_{\uparrow \downarrow}(p, q), \quad\left\{ \pm 1, \pm e^{1 \ldots n}\right\}
$$

ad $: \operatorname{Pin}_{\downarrow}(p, q) \rightarrow \mathrm{SO}(p, q), \quad$ ad $: \operatorname{Spin}_{\uparrow \downarrow}(p, q) \rightarrow \mathrm{SO}_{\uparrow \downarrow}(p, q), \quad\{ \pm 1\} ;$ 
4) если $p$ - нечётное, $q$ - чётное, то следующие гомоморфизмы сюргективны с соответствующим ядром:

$$
\operatorname{ad}: \operatorname{Pin}_{\downarrow}(p, q) \rightarrow \mathrm{SO}_{\uparrow \downarrow}(p, q), \quad\left\{ \pm 1, \pm e^{1 \ldots n}\right\}
$$

$$
\operatorname{ad}: \operatorname{Pin}_{\uparrow}(p, q) \rightarrow \mathrm{SO}(p, q), \quad \operatorname{ad}: \operatorname{Spin}_{\uparrow \downarrow}(p, q) \rightarrow \mathrm{SO}_{\uparrow \downarrow}(p, q), \quad\{ \pm 1\} .
$$

Доказ ат ель ст в о. Теорема следует из предыдущей теоремы о норме элементов спинорных групп.

Отметим, что в предыдущих утверждениях мы говорили о сюръективных отображениях с ядром $\{ \pm 1\}$, однако, более того, можно утверждать о двулистных накрытиях ортогональных групп спинорными (требуется проверить топологические свойства рассматриваемых групп).

Как мы видим, в случае нечётного $n$ гомоморфизм ad уже не описывает двулистное накрытие ортогональных групп спинорными. Ядро отображения в некоторых случаях состоит из 4 элементов. Например, возьмём произвольный элемент $t \in \operatorname{Pin}(p, q)$. Тогда ему очевидно ставится в соответствие та же ортогональная матрица, что и элементам $-t, e^{1 \ldots n} t,-e^{1 \ldots n} t$ в силу формулы $T e^{a} T^{-1}=p_{b}^{a} e^{b}$.

Следующая таблица отображает образ группы $\operatorname{Pin}(p, q)$ и ее компонент

\begin{tabular}{|c|c|c|c|c|c|}
\hline & $\mathrm{ad}$ & \multicolumn{4}{|c|}{$\mathrm{ad}$} \\
\hline & $(p, q)$ - любые & $\begin{array}{l}p-\text {-чётн. } \\
q-\text { чётн. }\end{array}$ & $\begin{array}{c}p-\text { - нечёт. } \\
q-\text { нечётн. }\end{array}$ & $\begin{array}{c}p-\text { нечётн. } \\
q-\text { чётн. }\end{array}$ & $\begin{array}{c}p-\text { чётн. } \\
q-\text { нечётн. }\end{array}$ \\
\hline $\operatorname{Pin}_{\uparrow}^{\prime}$ & $\mathrm{O}_{\uparrow}^{\prime}$ & $\mathrm{O}_{\uparrow}^{\prime}$ & $\mathrm{O}_{\downarrow}^{\prime}$ & $\mathrm{SO}^{\prime}$ & $\mathrm{SO}_{\uparrow \downarrow}$ \\
\hline $\operatorname{Pin}_{\downarrow}^{\prime}$ & $\mathrm{O}_{\downarrow}^{\prime}$ & $\mathrm{O}_{\downarrow}^{\prime}$ & $\mathrm{O}_{\uparrow}^{\prime}$ & $\mathrm{SO}_{\uparrow \downarrow}$ & $\mathrm{SO}^{\prime}$ \\
\hline Spin $^{\prime}$ & $\mathrm{SO}^{\prime}$ & $\mathrm{SO}^{\prime}$ & $\mathrm{SO}^{\prime}$ & $\mathrm{SO}^{\prime}$ & $\mathrm{SO}^{\prime}$ \\
\hline $\operatorname{Spin}_{\uparrow \downarrow}$ & $\mathrm{SO}_{\uparrow \downarrow}$ & $\mathrm{SO}_{\uparrow \downarrow}$ & $\mathrm{SO}_{\uparrow \downarrow}$ & $\mathrm{SO}_{\uparrow \downarrow}$ & $\mathrm{SO}_{\uparrow \downarrow}$ \\
\hline & & & & & \\
\hline
\end{tabular}
при действии гомоморфизмов ad и ad в случае различных сигнатур $(p, q)$ :

Заметим, что для построения общей картины связи спинорных и ортогональных групп удобно пользоваться измененным присоединенным представлением ad, которое ставит в соответствие спинорным группам одни и те же соответствующие ортогональные группы для случая всех сигнатур $(p, q)$. Вместе с тем, в частных случаях часто пользуются отображением ad, т. к. оно устроено проще. Например, в случае сигнатуры $(1,3)$ можно пользоваться обоими отображениями, но помнить, что при смене одного отображения на другое меняются местами накрытия ортохронной и ортохорной групп.

Автор выражает благодарность Н. Г. Марчуку за постановку задачи и полезные замечания. Работа выполнена при поддержке гранта Президента РФ (НШ-2928.2012.1).

\section{БИБЛИОГРАФИЧЕСКИЙ СПИСОК}

1. Д. С. Широков, "Обобщение теоремы Паули на случай алгебр Клиффорда" // Докл. Акад. наук, 2011. Т. 440, № 5. С. 607-610; англ. пер.: D. S. Shirokov, "Extension of Pauli's theorem to Clifford algebras" // Dokl. Math., 2011. Vol. 84, no. 2. Pp. 699-701.

2. Д. С. Широков, "Теорема о норме элементов спинорных групп" // Вестн. Сам. гос. техн. ун-та. Сер. Физ.-мат. науки, 2011. №1(22). С. 165-171. [D. S. Shirokov, "Theorem 
on the norm of elements of spinor groups"// Vestn. Samar. Gos. Tekhn. Univ. Ser. Fiz.-Mat. Nauki, 2011. no. 1(22). Pp. 165-171].

3. I. M. Benn, R. W. Tucker, An introduction to spinors and geometry with applications in physics. Bristol: Adam Hilger, Ltd., 1987. x+358 pp.

4. N. G. Marchuk, D. S. Shirokov, "Unitary spaces on Clifford algebras" // Adv. Appl. Clifford Algebr., 2008. Vol. 18, no. 2. Pp. 237-254.

Поступила в редакцию 16/XI/2012;

в окончательном варианте - 27/I/2013.

MSC: $15 \mathrm{A66}$

\section{THE USE OF THE GENERALIZED PAULI'S THEOREM FOR ODD ELEMENTS OF CLIFFORD ALGEBRA TO ANALYZE RELATIONS BETWEEN SPIN AND ORTHOGONAL GROUPS OF ARBITRARY DIMENSIONS}

\section{S. Shirokov}

Steklov Mathematical Institute, Russian Academy of Sciences,

8, Gubkina st., Moscow, 119991, Russia.

E-mail: shirokov@mi.ras.ru

In the present paper we consider the use of generalized Pauli's theorem to prove the theorem about double cover of orthogonal groups by spin groups. We prove theorems about double cover of orthochronous, othochorous, special and special orthochronous groups by corresponding spin groups. We show the difference between the approaches using adjoint action and twisted adjoint action.

Key words: Clifford algebra, Pauli's theorem, spin groups, orthogonal groups, double cover, orthochronous group, orthochorous group.

Original article submitted 16/XI/2012; revision submitted $27 / \mathrm{I} / 2013$.

Dmitry S. Shirokov, Postgraduate Student, Dept. of Mathematical Physics. 